\title{
Zero-Correlation Linear Cryptanalysis of Reduced-Round LBlock
}

\author{
Hadi Soleimany and Kaisa Nyberg \\ Aalto University School of Science, \\ Department of Information and Computer Science
}

\begin{abstract}
Zero-correlation linear attack is a new method for cryptanalysis of block ciphers developed by Bogdanov et al. in 2012. In this paper we adapt the matrix method to find zerocorrelation linear approximations. Then we present several zero-correlation linear approximations for 14 rounds of LBlock and describe a cryptanalysis for 22 rounds of the reduced LBlock. After biclique attacks on LBlock revealed weaknesses in its key schedule, its designers presented a new version of the cipher with a revised key schedule. The attack presented in this paper is applicable to LBlock structure independently of the key scheduling. The attack needs distinct known plaintexts which is a more realistic attack model in comparison with impossible differential cryptanalysis which uses chosen plaintext pairs. Moreover, we performed simulations on a small variant LBlock and present the first experimental results on the theoretical model of the multidimensional zero-correlation linear cryptanalysis method.
\end{abstract}

Keywords: block cipher, zero-correlation linear cryptanalysis, LBlock, matrix method

\section{Introduction}

Linear cryptanalysis is one of the most prominent cryptanalysis methods against block ciphers. Several extensions of linear cryptanalysis have been introduced which usually exploit several linear approximations with high correlation simultaneously. Kaliski and Robshaw used multiple linear approximations with the same key mask [7]. The concept of linear hull presented by Nyberg which uses several linear approximation with the same input and output masks [13]. Multiple linear approximations cryptanalysis and multidimensional linear cryptanalysis are proposed in [2] and [6] respectively. Recently, a novel extension of linear cryptanalysis was proposed which uses zero-correlation linear approximations [4]. It can be seen as the counterpart of impossible differential cryptanalysis. The original proposal had the disadvantage to require almost the full codebook of data. Bogdanov et.al. proposed a framework which uses several independent zero-correlation linear approximations to reduce data complexity [5]. Based on the multidimensional linear attack, a new distinguisher was recently proposed to eliminate the independence assumption [3]. The distinguisher is supposed to use distinct known plaintexts.

In this paper the multidimensional zero-correlation linear method is applied to attack 22 rounds of LBlock [16]. LBlock is a lightweight block cipher with semi-Feistel structure. The security of the cipher has been evaluated in $[8,11,16]$. The designers proposed integral and impossible differential cryptanalysis up to 20 rounds of LBlock. Using low diffusion of key schedule, an improved impossible differential cryptanalysis has been applied up to 22 rounds of LBlock [8]. The attack uses $2^{58}$ chosen plaintexts and the time complexity is $2^{79.28}$ which is almost equivalent to the exhaustive search. Since LBlock had been designed before biclique cryptanalysis introduction, the designers re-evaluate the security of LBlock and showed that the cipher is vulnerable against biclique cryptanalysis. They proposed a modified key schedule algorithm to improve the security of LBlock [15] such that the previous attacks are not applicable. In this paper, we show how to use the matrix method $[9,10]$ to find $8 \times 8$ different classes of zero-correlation linear approximations for 14 rounds which each one includes $2^{8}-1$ different zero-correlation approximations. Based on $2^{8}-1$ zero-correlation approximations we present an attack on 22 rounds of the reduced LBlock. This attack does not depend on the key schedule and it is applicable to both versions of LBlock. It exploits weaknesses in the permutation layer of LBlock to decrease the time complexity. The attack uses distinct known plaintexts. As depicted in Table 1, there is a trade-off between the time complexity and the data complexity of the attack.

The paper is structured as follows: In Section 2, we briefly describe LBlock. In Section 3 we review the previous work on zero-correlation linear cryptanalysis. In Section 4 we show how to use the matrix method as an automatic tool to find zero-correlation approximations and obtain several zero-correlation linear distinguisher for 14 rounds of the LBlock. Section 5 describes an attack on 22 rounds of LBlock. We conclude the paper in Section 6. 
Table 1. Summary of the Attacks on LBlock

\begin{tabular}{|c|c|c|c|c|c|}
\hline Attack & Rounds & Data & Time & Memory (Bytes) & Source \\
\hline Integral Attack (CP) & 20 & $2^{63.7}$ & $2^{63.7}$ & Not Specified & {$[16]$} \\
Impossible Differential (CP) & 20 & $2^{63}$ & $2^{72.7}$ & $2^{60}$ & {$[16]$} \\
Impossible Differential $^{\dagger}(\mathrm{CP})$ & 21 & $2^{62.5}$ & $2^{73.7}$ & $2^{64}$ & {$[11]$} \\
Impossible Differential $^{\dagger}(\mathrm{CP})$ & 21 & $2^{63}$ & $2^{69.5}$ & $2^{68}$ & {$[8]$} \\
Impossible Differential $^{\dagger}(\mathrm{CP})$ & 22 & $2^{58}$ & $2^{79.28}$ & $2^{68}$ & {$[8]$} \\
Zero Correlation (DKP) & 22 & $2^{64}$ & $2^{70.54}$ & $2^{64}$ & This paper \\
Zero Correlation (DKP) & 22 & $2^{62.1}$ & $2^{71.27}$ & $2^{64}$ & This paper \\
Zero Correlation (DKP) & 22 & $2^{60}$ & $2^{79}$ & $2^{64}$ & This paper \\
Biclique (KP) & Full & $2^{52}$ & $2^{78.4}$ & Negligible & {$[15]$} \\
\hline
\end{tabular}

$\dagger-$ this attack is applicable just on old version of the cipher, CP - Chosen Plaintexts, DKP - Distinct Known Plaintexts, KP - Known Plaintext

\section{A Brief Description of LBlock}

\section{$2.1 \quad$ Notation}

Throughout this paper we use the following notations:

$-S k_{i}: 32$-bit round key

- $i: i$-bit left cyclic shift

- $X(i): i$-th nibble of $X$ where the right most one is 0

$-X(i-j)$ : concatenation of $i, i-1, \cdots, j$-th nibble of $X$ where $i \geq j$

- $\mid$ : concatenation of two binary strings

$-L_{i} \mid R_{i}:$ the output of the $i$-round of LBlock

\subsection{LBlock Description}

LBlock is a variant Feistel block cipher with 32 rounds. It supports 80 secret key bits and the block size is $b=64$ bits. Each round includes 8 different $4 \times 4$ S-boxes and simple nibble-wise permutation. One round of LBlock and the round function are depicted in Figure 1.
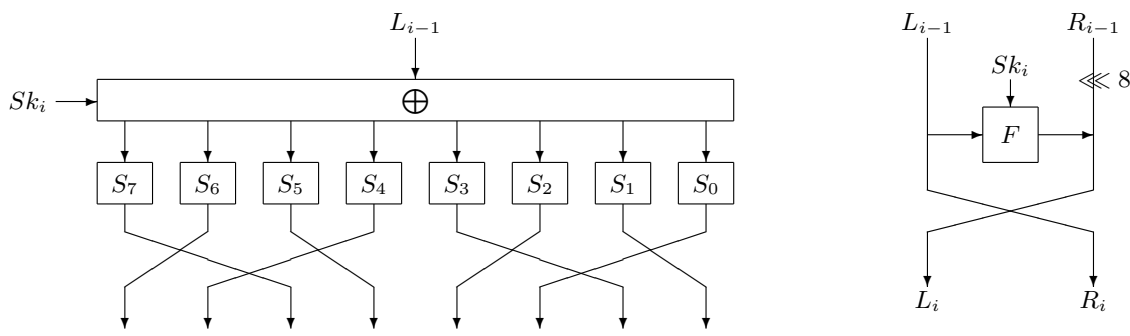

Fig. 1. One round of LBlock

Encryption Algorithm Let $P=L_{0} \mid R_{0}$ be a 64-bit plaintext. Then encryption process is as follows:

- For $i=1,2, \cdots, 31$, do

- $R_{i}=L_{i-1}$

- $L_{i}=F\left(L_{i-1}, S K_{i}\right) \oplus\left(R_{i-1} \lll 8\right)$

$-L_{32}=L_{31}, R_{32}=F\left(L_{31}, S K_{32}\right) \oplus\left(R_{31} \lll 8\right)$

$-C=L_{32} \mid R_{32}$.

In our attacks on reduced-round LBlock we also consider the last round to be without swapping the halves as in the original LBlock.

Key schedule The 80-bit master key $K$ is stored in a key register. In the $i$-th step, the leftmost 32 bits of current content of register $K$ are extracted as the round key $S K_{i}$. Then the key register is updated in each round. We do not exploit any property of the key scheduling. For the updating procedure in the original LBlock and the new version we refer to [16] and [15], respectively. 


\section{Zero-Correlation Linear Approximation}

Consider a function $f: \mathbb{F}_{2}^{n} \mapsto \mathbb{F}_{2}^{m}$ and let the input of the function be $x \in \mathbb{F}_{2}^{n}$. A linear approximation with an input mask $u$ and an output mask $v$ is the following function:

$$
x \mapsto u \cdot x \oplus v \cdot f(x) .
$$

The linear approximation has probability

$$
p(u ; v)=\operatorname{Pr}(u \cdot x \oplus v \cdot f(x)=0)
$$

and its correlation is defined as follows:

$$
c_{f}(u ; v)=2 p(u ; v)-1
$$

In linear cryptanalysis we are interested in the linear approximation with correlation far from zero. The number of known plaintexts needed in the linear cryptanalysis is inversely proportional to the squared correlation. Zero-correlation linear cryptanalysis uses linear approximations such that the correlation is equal to zero for all keys. If the number of zero-correlation approximations is $2^{m}$, then by [3] the number of required distinct plaintexts is about $2^{n+2-m / 2}$. The key recovery can be done with the same method utilized by Matsui's Algorithm 2 [12].

To describe this process in more detail, let us describe a cipher $E$ as a cascade $E=E_{f} \circ E_{z} \circ E_{b}$. Assume there exists $m$ independent linear approximations for $E_{z}$ such that all $\ell=2^{m}-1$ nonzero linear combinations of them have correlation zero. For each key candidate, the adversary encrypts the plaintexts for the beginning rounds $E_{b}$ and decrypts the corresponding ciphertexts for the final rounds $E_{f}$.

For each of $i \in \mathbb{F}_{2}^{m}$ he allocates a counter $T_{i}$ and computes the number of times which the corresponding data value is equal to $i$. Then the adversary computes the statistic $T$ value

$$
T=\sum_{i=0}^{2^{m}-1} \frac{\left(T_{i}-N 2^{-m}\right)^{2}}{N 2^{-m}\left(1-2^{-m}\right)}
$$

The value $T$ for right key guess follows a $\chi^{2}$-distribution with mean $\mu_{0}=\ell \frac{2^{n}-N}{2^{n}-1}$ and variance $\sigma_{0}^{2}=2 \ell\left(\frac{2^{n}-N}{2^{n}-1}\right)^{2}$ while for the wrong key the distribution is a $\chi^{2}$-distribution with mean $\mu_{1}=\ell$ and variance $\sigma_{1}^{2}=2 \ell$.

Let show error probability type I as $\alpha$ and error probability type II as $\beta$. If we consider the decision threshold $t=\mu_{0}+\sigma_{0} z_{1-\alpha}=\mu_{1}+\sigma_{1} z_{1-\beta}$ then the amount of distinct known plaintexts is as follows:

$$
N=\frac{2^{n}\left(z_{1-\alpha}+z_{1-\beta}\right)}{\sqrt{\ell / 2}-z_{1-\beta}}
$$

where $z_{p}=\Phi^{-1}(p)$ for $0<p<1$ where $\Phi$ is the cumulative function of the standard normal distribution. For more details we refer to [3].

\section{The Matrix Method}

Several tools have been proposed for finding statistical distinguisher. Such tools help us to analyze algorithms systematically. A cryptanalytic tool for finding impossible differential characteristics in block ciphers with bijective function was introduced in $[9,10]$. It is called the matrix method and uses the "miss-in-the-middle" approach to find impossible differential characteristic. The miss-in-the-middle technique proposes to construct the impossible differential characteristic by two (truncated) differential paths with probability one and which lead to a contradiction in the middle. The matrix method is a tool for finding these paths. In this section we show this technique is also useful for finding zero-correlation linear approximation. We can follow the linear approximation patterns of input and output masks in the intermediate rounds and inquire whether no linear characteristics with non-zero-correlation exists. So the matrix method is also useful to automate the process of finding the longest zero-correlation linear approximations. 


\subsection{Matrix Method for Finding Linear Approximation with Correlation Zero}

The state is partitioned into $n$ words (usually of the same length). In the linear approximation, the linear masks applied to the words can be of the following five types:

1. zero mask, denoted by 0 ,

2. an arbitrary non-zero mask, denoted by $\overline{0}$,

3. non-zero mask with a fixed value $a$, denoted by $a$,

4. the exclusive-or of a fixed non-zero mask $a$ and an arbitrary non-zero mask, denoted by $\bar{a}$,

5 . any mask, denoted by $*$.

After that we describe the encryption round as a matrix $M^{n \times n}$. The matrix shows how a linear mask of each output word is affected by the linear mask of an input word. Let show the input and the output of the round by two bit strings $A$ and $B$ respectively. If $B(j)$ is not affected by a linear mask of $A(i)$ the value $(i, j)$ set to 0 . If a linear mask of $A(i)$ affects $B(j)$ directly the value $(i, j)$ set to 1 . Finally if $B(j)$ is affected by a linear mask of $A(i)$ after the round function the value $(i, j)$ set to $1_{F}$. For decryption of the round, another matrix is defined similarly. To define the matrices we can use lemmas in Appendix A which are introduced in [4] (see also [1]).

We can apply the exclusive-or operation to the five types of masks defined above resulting in arithmetic rules. These rules are given in the table on the left of Table 2. On the right hand side of Table 2 we show how certain operations will modify the five types of masks. The operations are: multiplication by 0 , multiplication by 1 and multiplication by $1_{F}$. The last operation means that, given a bijection $F$ and a type $\tau$ of masks, $\tau \cdot 1_{F}$ is the type of masks $v$ for which there is $u$ of type $\tau$ such that the linear approximation $u \cdot x+v \cdot F(x)=0$ holds with non-zero correlation.

Table 2. Arithmetic rules. The table of the left gives the addition rules between two mask types. The table on the right shows the operation rules of multiplication by 0,1 and $1_{F}$.

\begin{tabular}{|c|ccccc|}
\hline+ & 0 & $\overline{0}$ & $a$ & $\bar{a}$ & $*$ \\
\hline 0 & 0 & $\overline{0}$ & $a$ & $\bar{a}$ & $*$ \\
$\overline{0}$ & $\overline{0}$ & $*$ & $\bar{a}$ & $*$ & $*$ \\
$b$ & $b$ & $\bar{b}$ & $a+b$ & $*$ & $*$ \\
$\bar{b}$ & $\bar{b}$ & $*$ & $*$ & $*$ & $*$ \\
$*$ & $*$ & $*$ & $*$ & $*$ & $*$ \\
\hline
\end{tabular}

\begin{tabular}{|c|ccc|}
\hline & 0 & 1 & $1_{F}$ \\
\hline & & & \\
0 & 0 & 0 & 0 \\
$\overline{0}$ & 0 & $\overline{0}$ & $\overline{0}$ \\
$a$ & 0 & $a$ & $\overline{0}$ \\
$\bar{a}$ & 0 & $\bar{a}$ & $*$ \\
$*$ & 0 & $*$ & $*$ \\
\hline
\end{tabular}

For a given state, we use the matrix iteratively to obtain the new state over multiple rounds. To find the longest zero-correlation linear approximation we compute the new states in both forward and backward directions just before the values of all words become only $*$. Finally, we scan intermediate values and check the incoherence of events.

For example the encryption matrix of Feistel structure is $\left(\begin{array}{cc}0 & 1 \\ 1 & 1\end{array} 1_{F}\right)$. If we assume the initial mask type as $(a, 0)$ the mask type of the third round can be obtained as follows:

$$
\begin{aligned}
& (a, 0) \cdot\left(\begin{array}{ll}
0 & 1 \\
1 & 1_{F}
\end{array}\right)=\left(a \cdot 0+0 \cdot 1, a \cdot 1+0 \cdot 1_{F}\right)=(0+0, a+0)=(0, a) \\
& (0, a) \cdot\left(\begin{array}{ll}
0 & 1 \\
1 & 1_{F}
\end{array}\right)=\left(0 \cdot 0+a \cdot 1,0 \cdot 1+a \cdot 1_{F}\right)=(0+a, 0+\overline{0})=(a, \overline{0}) \\
& (a, 1) \cdot\left(\begin{array}{ll}
0 & 1 \\
1 & 1_{F}
\end{array}\right)=\left(a \cdot 0+\overline{0} \cdot 1, a \cdot 1+\overline{0} \cdot 1_{F}\right)=(0+\overline{0}, a+\overline{0})=(\overline{0}, \bar{a})
\end{aligned}
$$

\subsection{Zero-Correlation Linear Approximation for 14-rounds of LBlock}

We applied the matrix method for LBlock. The round matrices for encryption and decryption can be found in Appendix B. The longest zero-correlation linear approximation was obtained for 14 rounds of LBlock. If the input mask would be exactly one non-zero nibble in $L_{r}$ and the output mask after 14 
rounds would be one non-zero nibble in $R_{r+14}$, then the linear approximation has correlation zero. For example $(000 a 0000 \mid 00000000) \rightarrow(00000000 \mid 06000000)$ has correlation exactly zero which the values $a$ and $b$ are non zero. The development of the states of mask types as encryption proceeds from round to round is depicted in Table 3 . The contradiction occurs in $R_{7}(5)$. We note there exists $8 \times 8$ different classes of zero-correlation linear approximations for 14 rounds each of which includes $2^{8}-1$ different zero-correlation approximations. We will use this observation to build a multidimensional linear approximation to minimize the data complexity as described in Section 3.

Table 3. Zero-correlation linear approximation for 14-round LBlock

\begin{tabular}{|c|c|c|}
\hline Round & $\Gamma_{L_{r}}$ & $\Gamma_{R_{r}}$ \\
\hline 0 & $000 a 0000$ & 00000000 \\
1 & 00000000 & $000 a 0000$ \\
2 & $0 a 000000$ & $000 \overline{0} 0000$ \\
3 & $0 \overline{0} 000000$ & $0 a 0 \overline{0} 0000$ \\
4 & $0 \overline{0} 00000 a$ & $0 \overline{0} 0 \overline{0} 00 \overline{0} 0$ \\
5 & $0 \overline{0} 00 \overline{0} 00 \overline{0}$ & $0 \overline{0} 0 \overline{0} 0 \overline{0} \overline{0} a$ \\
6 & $0 \overline{0} 0 \overline{0} \overline{0} a 0 \overline{0}$ & $0 \overline{0} \overline{0} \overline{0} \overline{0} \overline{0} \overline{0} *$ \\
7 & $\overline{0} \overline{0} \overline{0} \overline{0} \overline{0} * 0 \overline{0}$ & $\overline{0} * \overline{0} * \overline{0} \bar{a} \overline{0} *$ \\
\hline \hline 7 & $\overline{0} \overline{0} * * \overline{0} \overline{0} * \overline{0}$ & $\overline{0} * 0 \overline{0} \overline{0} \overline{0} \overline{0} \overline{0}$ \\
8 & $0 \overline{0} \overline{0} \overline{0} \overline{0} \overline{0} *$ & $0 \overline{0} \overline{0} \overline{0} 00 \overline{0} b$ \\
9 & $\overline{0} \overline{0} 00 \overline{0} b 0 \overline{0}$ & $00 \overline{0} 0 \overline{0} 00 \overline{0}$ \\
10 & $\overline{0} 0 \overline{0} 00 \overline{0} 00$ & $0000 \overline{0} b 00$ \\
11 & $00 \overline{0} b 0000$ & $00000 \overline{0} 00$ \\
12 & $000 \overline{0} 0000$ & $000 b 0000$ \\
13 & $0 b 000000$ & 00000000 \\
14 & 00000000 & $0 b 000000$ \\
\hline
\end{tabular}

\section{Zero-Correlation Linear Cryptanalysis of 22 Reduced-Round LBlock}

In this section, we propose a zero-correlation linear attack on 22-round LBlock. The attack utilizes the 14-round zero-correlation linear approximations described in Table 3 from round 5 to 18. After collecting sufficient plaintext-ciphertext pairs, we guess corresponding subkeys for the first four rounds and the last four rounds and estimate the correlation of approximations as described in Algorithm 1 in Appendix C.

Based on the error probabilities $\alpha$ and $\beta$, the number of pairs $N$ in Algorithm 1 and the decision threshold $t$ are determined. The time complexity of the Algorithm 1 is $N \cdot 2^{28} \cdot 2^{28}$ where $N$ is the number of plaintexts used in the cryptanalysis. So the time complexity is much more than exhaustive search. To overcome this restriction we note $L_{4}(4)$ and $R_{18}(6)$ are not affected by all bits in rounds $1-4$ and $19-22$. So Algorithm 1 is not optimal and it repeats the same procedure for different pairs. We show that it is possible to remove repetitions and reduce time complexity significantly.

The nibble $L_{4}(4)$ is affected by 32 bits of plaintext $L_{0} \mid R_{0}, 20$ bits of $L_{1} \mid R_{1}, 12$ bits of $L_{2} \mid R_{2}$ and 8 bits of $L_{3} \mid R_{3}$. Also $L_{18}(6)$ is affected by 32 bits of ciphertext $L_{22} \mid R_{22}, 20$ bits of $L_{21} \mid R_{21}, 12$ bits of $L_{20} \mid R_{20}$ and 8 bits of $L_{19} \mid R_{19}$. We call these bits "active" and other ones "neutral". The idea is to ignore neutral bits and instead of encrypting and decrypting all plaintex-ciphertext pairs, do it only once and count the number of pairs, which have the same value in active bits. In each step, for each subkey candidate, we encrypt (decrypt) active bits in round $r$ over one round and count the number of pairs which give the same value in active bits in round $r+1(r-1)$.

The attack procedure is as follows:

1. Collect $N$ plaintexts with corresponding ciphertexs.

2. Allocate a 8-bit counter $N_{0}\left[x_{0}, x_{22}\right]$ for each of $2^{64}$ possible values of $\left(x_{0} \mid x_{22}\right)$ where $x_{0}=$ $L_{0}(5,4,2,1,0) \mid R_{0}(6,4,1)$ and $x_{22}=L_{22}(7,6,4,2,0) \mid R_{22}(7,5,2)$ and set them zero. Calculate the number of pairs of plaintext-ciphertext with given values $x_{0}$ and $x_{22}$ and save it in $N_{0}\left[x_{0}, x_{22}\right]$. In this step, around $2^{64}$ plaintext-ciphertext pairs are divided into $2^{64}$ different state. The expected pairs for each state is around one. So the assumption $N_{0}$ as a 8-bit counter is sufficient. 
3. Guess the 3 nibbles $S K_{1}(4,2,1)$. Allocate a counter $N_{1}\left[x_{1}, x_{22}\right]$ for each of $2^{52}$ possible values of $\left(x_{1} \mid x_{22}\right)$ where $x_{1}=L_{1}(6,3,0) \mid R_{1}(5,0)$ and set them zero. For all $2^{32}$ possible values of $x_{0}$, encrypt $x_{0}$ one round to obtain $x_{1}$ and update the value $N_{1}\left[x_{1}, x_{22}\right]=N_{1}\left[x_{1}, x_{22}\right]+N_{0}\left[x_{0}, x_{22}\right]$ for all $2^{32}$ values of $x_{22}$

4. Guess 2 nibbles $S K_{2}(6,0)$. Allocate a counter $N_{2}\left[x_{2}, x_{22}\right]$ for each of $2^{44}$ possible values of $\left(x_{2} \mid x_{22}\right)$ where $x_{2}=L_{2}(7,2) \mid R_{2}(3)$ and set them zero. For all $2^{20}$ possible values of $x_{1}$, encrypt $x_{1}$ one round to obtain $x_{2}$ and update the value $N_{2}\left[x_{2}, x_{22}\right]=N_{2}\left[x_{2}, x_{22}\right]+N_{1}\left[x_{1}, x_{22}\right]$ for all $2^{32}$ values of $x_{22}$.

5. Guess the nibble $S K_{3}(7)$. Allocate a counter $N_{3}\left[x_{3}, x_{22}\right]$ for each of $2^{40}$ possible values of $\left(x_{3} \mid x_{22}\right)$ where $x_{3}=L_{3}(5) \mid R_{3}(2)$ and set them zero. For all $2^{12}$ possible values of $x_{2}$, encrypt $x_{2}$ one round to obtain $x_{3}$ and update the value $N_{3}\left[x_{3}, x_{22}\right]=N_{3}\left[x_{3}, x_{22}\right]+N_{2}\left[x_{2}, x_{22}\right]$ for all $2^{32}$ values of $x_{22}$.

6. Guess the nibble $S K_{4}(5)$. Allocate a counter $N_{4}\left[x_{4}, x_{22}\right]$ for each of $2^{36}$ possible values of $\left(x_{4} \mid x_{22}\right)$ where $x_{4}=L_{4}(4)$ and set them zero. For all $2^{8}$ possible values of $x_{3}$, encrypt $x_{3}$ one round to obtain $x_{4}$ and update the value $N_{4}\left[x_{4}, x_{22}\right]=N_{3}\left[x_{4}, x_{22}\right]+N_{3}\left[x_{3}, x_{22}\right]$ for all $2^{32}$ values of $x_{22}$.

7. Guess the 3 nibbles $S K_{22}(7,6,0)$. Allocate a counter $N_{5}\left[x_{4}, x_{21}\right]$ for each of $2^{24}$ possible values of $\left(x_{4} \mid x_{21}\right)$ where $x_{21}=L_{21}(4,2) \mid R_{21}(5,3,0)$ and set them zero. For all $2^{32}$ possible values of $x_{22}$, decrypt $x_{22}$ one round to obtain $x_{21}$ and update the value $N_{5}\left[x_{4}, x_{21}\right]=N_{5}\left[x_{4}, x_{21}\right]+N_{4}\left[x_{4}, x_{22}\right]$ for all $2^{4}$ values of $x_{4}$.

8. Guess 2 nibbles $S K_{21}(5,0)$. Allocate a counter $N_{6}\left[x_{4}, x_{20}\right]$ for each of $2^{16}$ possible values of $\left(x_{4} \mid x_{20}\right)$ where $x_{20}=L_{20}(3) \mid R_{20}(2,0)$ and set them zero. For all $2^{20}$ possible values of $x_{21}$, decrypt $x_{21}$ one round to obtain $x_{20}$ and update the value $N_{6}\left[x_{4}, x_{20}\right]=N_{6}\left[x_{4}, x_{20}\right]+N_{5}\left[x_{4}, x_{21}\right]$ for all $2^{4}$ values of $x_{4}$.

9. Guess the nibble $S K_{20}(2)$. Allocate a counter $N_{7}\left[x_{4}, x_{19}\right]$ for each of $2^{12}$ possible values of $\left(x_{4} \mid x_{19}\right)$ where $x_{19}=L_{19}(0) \mid R_{19}(1)$ and set them zero. For all $2^{12}$ possible values of $x_{20}$ decrypt $x_{20}$ one round to obtain $x_{19}$ and update the value $N_{7}\left[x_{4}, x_{19}\right]=N_{7}\left[x_{4}, x_{19}\right]+N_{6}\left[x_{4}, x_{20}\right]$ for all $2^{4}$ values of $x_{4}$.

10. Guess the nibble $S K_{19}(1)$. Allocate a counter $N_{8}\left[x_{4}, x_{18}\right]$ for each of $2^{8}$ possible values of $\left(x_{4} \mid x_{18}\right)$ where $x_{18}=R_{18}(6)$ and set them zero. For all $2^{8}$ possible values of $x_{19}$, decrypt $x_{19}$ one round to obtain $x_{18}$ and update the value $N_{8}\left[x_{4}, x_{18}\right]=N_{8}\left[x_{4}, x_{18}\right]+N_{7}\left[x_{4}, x_{19}\right]$ for all $2^{4}$ values of $x_{4}$.

11. Compute the statistic value $T=N \cdot 2^{8} \sum_{x_{4}=0}^{2^{4}-1} \sum_{x_{18}=0}^{2^{4}-1}\left(\frac{N_{8}\left[x_{4}, x_{18}\right]}{N}-\frac{1}{2^{8}}\right)$. If $T<t$, then the guess key is a possible candidate.

12. Do exhaustive search for all keys which corresponds to the guess subkey bits.

\section{Attack complexity}

The memory complexity of the attack is dominated by step 2 which needs $2^{64}$ bytes. Time complexity of step 1 and 2 is equal to the number of needed plaintext-ciphertext pairs $N$. The time complexity in each step between 3 and 11 depends on the number of accesses to the memory. The time complexity for each round is listed seperately in Appendix D. Also step 12 requires $2^{80} \cdot \beta$ full encryption because we expect a wrong subkey survives with probability $\beta$.

The time complexity is dominated by step 3 and step 12 . The time complexity of round 3 is $2^{76}$ memory accesses. If we consider one memory accesses as a half round, the time complexity of step 3 is $2^{76} \times \frac{1}{2} \times \frac{1}{22}=2^{70.54}$ of 22 -round LBlock. Based on the error probability type I $\alpha$ and error probability type II $\beta$, the number of plaintexts-ciphetexts pairs needed, time complexity of step 12 and success probability are determined.

There is a trade-off between the time complexity and the data complexity of the attack, as depicted in Table 1. To reduce the time complexity as much as possible, we assume to have access the full codebook. In this case, the error probabilities and time complexity of step 12 is negligible compared to the complexity of step 3. To have a lowest data complexity, we can set $\alpha=2^{-2.7}$ and $\beta=2^{-1}$. In this case data complexity decreases to $N=2^{60}$ in cost of increasing time complexity. The time complexity is dominated by step 12 which needs $2^{80} \cdot 2^{-1}=2^{79} 22$-round LBlock encryption. The more realistic assumption is the state between these cases. For example, if we set $\alpha=2^{-2.7}$ and $\beta=2^{-10}$ then $z_{1-\alpha}=1$ and $z_{1-\beta}=3.09$. Equation $(2)$ determines the data complexity $N=2^{62.1}$. The time complexity is dominated by step 3 and $122^{70.5}+2^{70}=2^{71.27}$. The success probability is $1-\alpha=0.84$.

\section{Experimental Results}

As noted in [16] LBlock can be described as a 16-branch generalized Feistel cipher (GFC) with improved permutation. The nibble-wise permutation has been chosen such that it achieves the best diffusion as 
proposed in [14]. To verify the theoretical model of zero-correlation attacks [3] we implement the described attack on a small variant of LBlock with block length 32-bit. Two optimal word-wise permutations for improved Type II GFC with 8 branches were suggested in [14]. To make the small variant cipher similar as much as possible to the original one, we choose the permutation which is not based on the Bruijn graph. We consider the key schedule like original LBlock and choose the leftmost 16 bits of the register $K$ as the round key. Matrix method produces a similar multidimensional linear approximation with correlation zero for 10 rounds of the small variant of LBlock cipher. This linear approximation has exactly one non-zero nibble in the input mask and one non-zero nibble in the output mask.

To evaluate a 10-round distinguisher from round 2 to 12 , we consider 14 rounds of the small LBlock. The distinguisher depends on 2-nibbles of subkeys in the first two rounds and 2-nibbles of subkeys in the last two rounds. We consider 30 different sets of distinct known plaintexts with different secret keys. In each experiment the behavior of the statistic $T$ in Equation 1 is studied for the right key and also for (just) one wrong key. The results of the implementation is shown in Figure 2. As predicted by the theoretical model, when more than $2^{30.2}$ distinct known plaintexts are used, the correct key is very likely to pass the test, while the wrong keys would fail. Access to the full codebook leads to the key recovery with negligible error probability. When using $2^{28}$ distinct known plaintexts, the right key survives with high probability but several wrong keys remain too.

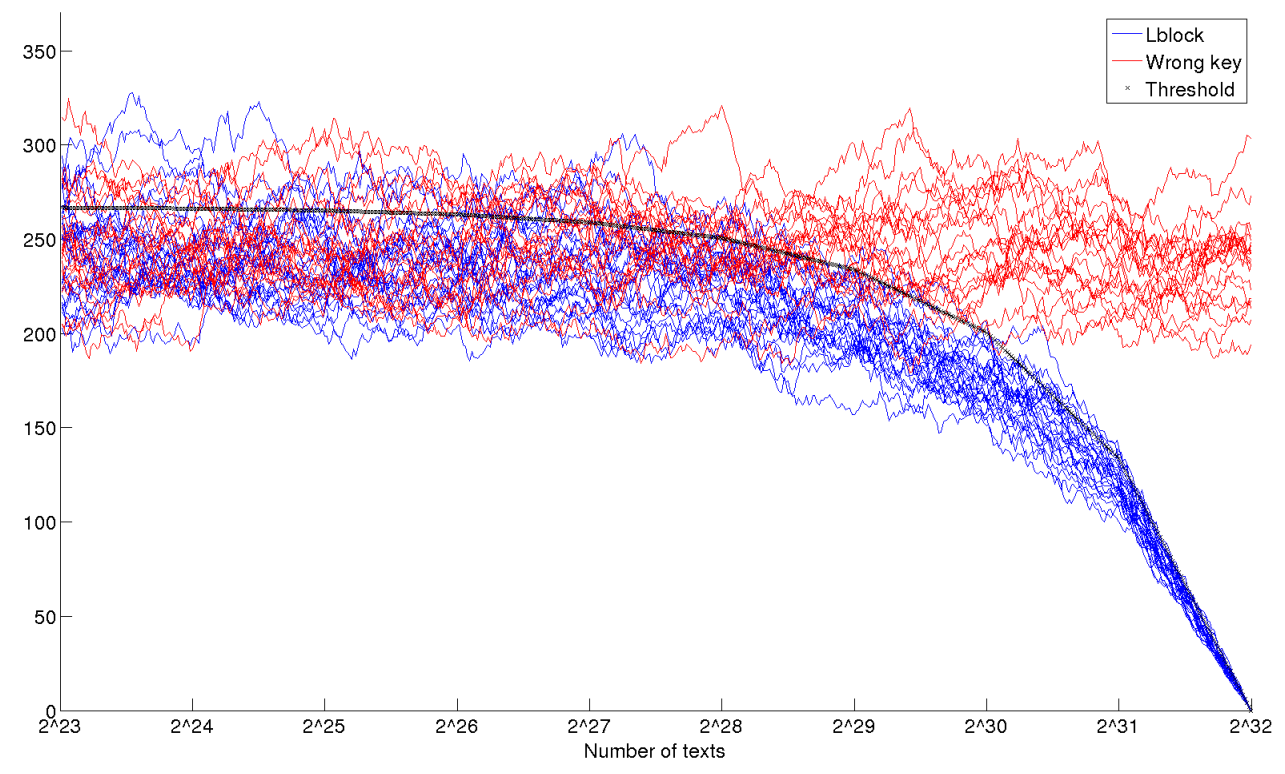

Fig. 2. Experimental results for small variant of LBlock

\section{Conclusion}

In this paper we showed how to use the matrix method to establish zero-correlation linear approximations automatically. We used this method to obtain several zero-correlation linear approximations over 14 rounds of LBlock. We believe that the described method will be useful for analysis of other block ciphers, too. Based on the 14-round distinguisher we present an attack on 22 rounds of LBlock. While the previous attack, which can break the same number of rounds, uses chosen plaintext pairs, our attack assumes only that the plaintexts are distinct. Unlike biclique techniques, the proposed cryptanalysis does not exploit the structure of the key schedule and therefore applies also to the new version of LBlock. Finally, we implement the attack for a small variant of LBlock and run simulations to experimentally validate the statistical model of zero-correlation linear cryptanalysis presented in [3]. 
Acknowledgments. The authors would like to thank Céline Blondeau and Risto Hakala for helpful comments and suggestions.

\section{References}

1. Biham, E.: On Matsui's Linear Cryptanalysis. In: Santis, A.D. (ed.) EUROCRYPT 1994. LNCS, vol. 950, pp. 341-355. Springer (1994)

2. Biryukov, A., Cannière, C.D., Quisquater, M.: On Multiple Linear Approximations. In: Franklin, M.K. (ed.) CRYPTO 2004. LNCS, vol. 3152, pp. 1-22. Springer (2004)

3. Bogdanov, A., Leander, G., Nyberg, K., Wang, M.: Integral and Multidimensional Linear Distinguishers with Correlation Zero. In: Wang, X., Sako, K. (eds.) ASIACRYPT 2012. LNCS, vol. 7658, pp. 244-261. Springer (2012)

4. Bogdanov, A., Rijmen, V.: Zero-correlation linear cryptanalysis of block ciphers. vol. 2011, p. 123 (2011)

5. Bogdanov, A., Wang, M.: Zero Correlation Linear Cryptanalysis with Reduced Data Complexity. In: Canteaut, A. (ed.) FSE 2012. LNCS, vol. 7549, pp. 29-48. Springer (2012)

6. Hermelin, M., Cho, J.Y., Nyberg, K.: Multidimensional Extension of Matsui's Algorithm 2. In: Dunkelman, O. (ed.) FSE 2009. LNCS, vol. 5665, pp. 209-227. Springer (2009)

7. Jr., B.S.K., Robshaw, M.J.B.: Linear Cryptanalysis Using Multiple Approximations. In: Desmedt, Y. (ed.) CRYPTO 1994. LNCS, vol. 839, pp. 26-39. Springer (1994)

8. Karakoç, F., Demirci, H., Harmanci, A.E.: Impossible Differential Cryptanalysis of Reduced-Round LBlock. In: Askoxylakis, I.G., Pöhls, H.C., Posegga, J. (eds.) WISTP 2012. LNCS, vol. 7322, pp. 179-188. Springer (2012)

9. Kim, J., Hong, S., Lim, J.: Impossible differential cryptanalysis using matrix method. Discrete Mathematics 310(5), 988-1002 (2010)

10. Kim, J., Hong, S., Sung, J., Lee, C., Lee, S.: Impossible Differential Cryptanalysis for Block Cipher Structures. In: Johansson, T., Maitra, S. (eds.) INDOCRYPT 2003. LNCS, vol. 2904, pp. 82-96. Springer (2003)

11. Liu, Y., Gu, D., Liu, Z., Li, W.: Impossible Differential Attacks on Reduced-Round LBlock. In: Ryan, M.D., Smyth, B., Wang, G. (eds.) ISPEC 2012. LNCS, vol. 7232, pp. 97-108. Springer (2012)

12. Matsui, M.: Linear Cryptoanalysis Method for DES Cipher. In: Helleseth, T. (ed.) EUROCRYPT 1993. LNCS, vol. 765, pp. 386-397. Springer (1994)

13. Nyberg, K.: Linear Approximation of Block Ciphers. In: Helleseth, T. (ed.) EUROCRYPT 1994. LNCS, vol. 950, pp. 439-444. Springer (1995)

14. Suzaki, T., Minematsu, K.: Improving the Generalized Feistel. In: Hong, S., Iwata, T. (eds.) FSE 2010. LNCS, vol. 6147, pp. 19-39. Springer (2010)

15. Wang, Y., Wu, W., Yu, X., Zhang, L.: Security on LBlock against Biclique Cryptanalysis. In: Lopez, J., Tsudik, G. (eds.) WISA 2012. LNCS, vol. 7690, pp. 1-14. Springer (2012)

16. Wu, W., Zhang, L.: LBlock: A Lightweight Block Cipher. In: Lopez, J., Tsudik, G. (eds.) ACNS 2011. LNCS, vol. 6715, pp. 327-344. Springer (2011)

\section{A Lemmas}

To describe the encryption or decryption round, we can use the following lemmas:

Lemma 1. XOR operation: Let $f\left(x_{1}, x_{2}\right)=x_{1} \oplus x_{2}$ then the correlation of linear approximation $u_{1} \cdot x_{1}+u_{2} \cdot x_{2}=v \cdot f\left(x_{1}, x_{2}\right)$ is non-zero if and only if $u_{1}=u_{2}=v$.

Lemma 2. Branching operation: Let $f(x)=(x, x)$ then the correlation of linear approximation $u_{1} \cdot x+u_{2} \cdot x=v \cdot f(x)$ is non-zero if and only if $u=v_{1}+v_{2}$.

Lemma 3. Bijective function: Let $f(x)$ be a bijective function then the correlation of linear approximation $u \cdot x=v \cdot f(x)$ is non-zero if and only if $u=v=0$ or $u \neq 0$ and $v \neq 0$. 


\section{B LBlock Matrices}

$$
M_{\text {Encryption }}=\left(\begin{array}{cccccccccccccccc}
0 & 0 & 0 & 0 & 0 & 0 & 0 & 0 & 1 & 0 & 0 & 0 & 0 & 0 & 0 & 0 \\
0 & 0 & 0 & 0 & 0 & 0 & 0 & 0 & 0 & 1 & 0 & 0 & 0 & 0 & 0 & 0 \\
0 & 0 & 0 & 0 & 0 & 0 & 0 & 0 & 0 & 0 & 1 & 0 & 0 & 0 & 0 & 0 \\
0 & 0 & 0 & 0 & 0 & 0 & 0 & 0 & 0 & 0 & 0 & 1 & 0 & 0 & 0 & 0 \\
0 & 0 & 0 & 0 & 0 & 0 & 0 & 0 & 0 & 0 & 0 & 0 & 1 & 0 & 0 & 0 \\
0 & 0 & 0 & 0 & 0 & 0 & 0 & 0 & 0 & 0 & 0 & 0 & 0 & 1 & 0 & 0 \\
0 & 0 & 0 & 0 & 0 & 0 & 0 & 0 & 0 & 0 & 0 & 0 & 0 & 0 & 1 & 0 \\
0 & 0 & 0 & 0 & 0 & 0 & 0 & 0 & 0 & 0 & 0 & 0 & 0 & 0 & 0 & 1 \\
0 & 0 & 0 & 0 & 0 & 0 & 1 & 0 & 0 & 0 & 0 & 0 & 1_{F} & 0 & 0 & 0 \\
0 & 0 & 0 & 0 & 0 & 0 & 0 & 1 & 0 & 0 & 0 & 0 & 0 & 0 & 1_{F} & 0 \\
1 & 0 & 0 & 0 & 0 & 0 & 0 & 0 & 0 & 1_{F} & 0 & 0 & 0 & 0 & 0 & 0 \\
0 & 1 & 0 & 0 & 0 & 0 & 0 & 0 & 0 & 0 & 0 & 1_{F} & 0 & 0 & 0 & 0 \\
0 & 0 & 1 & 0 & 0 & 0 & 0 & 0 & 1_{F} & 0 & 0 & 0 & 0 & 0 & 0 & 0 \\
0 & 0 & 0 & 1 & 0 & 0 & 0 & 0 & 0 & 0 & 1_{F} & 0 & 0 & 0 & 0 & 0 \\
0 & 0 & 0 & 0 & 1 & 0 & 0 & 0 & 0 & 0 & 0 & 0 & 0 & 1_{F} & 0 & 0 \\
0 & 0 & 0 & 0 & 0 & 1 & 0 & 0 & 0 & 0 & 0 & 0 & 0 & 0 & 0 & 1_{F}
\end{array}\right)
$$

\section{Basic Key Recovery Attack on 22 Reduced-Round LBlock}

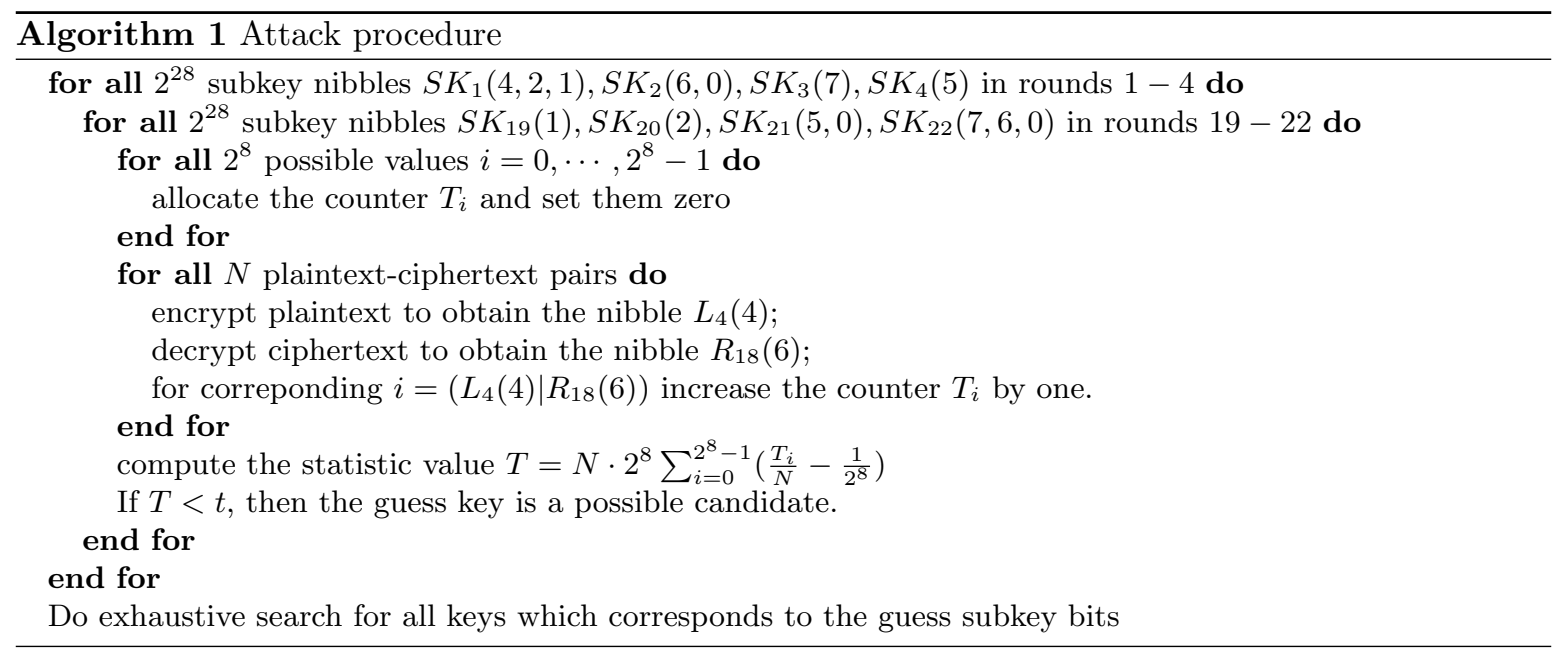

\section{Attack Complexity}

The time complexity of steps $3-11$ in the described attack in Section 5 is as follows: 
Step 3 requires $2^{12} \times 2^{32} \times 2^{32}=2^{76}$ memory accesses, because we should guess 12 bits for $S K_{1}$, and for $2^{32}$ values encrypt $x_{0}$ one round and then update $N_{1}$ for $2^{32}$ times.

Step 4 requires $2^{12} \times 2^{8} \times 2^{20} \times 2^{32}=2^{72}$ memory accesses, because for all of guessed $2^{12}$ keys in previous step, we should guess 8 bits for $S K_{2}$, and for $2^{20}$ values encrypt $x_{1}$ one round and then update $N_{2}$ for $2^{32}$ times.

Step 5 requires $2^{20} \times 2^{4} \times 2^{12} \times 2^{32}=2^{68}$ memory accesses, because for all of guessed $2^{20}$ keys in previous steps, we should guess 4 bits for $S K_{3}$ and for $2^{12}$ values encrypt $x_{2}$ one round and then update $N_{3}$ for $2^{32}$ times.

Step 6 requires $2^{24} \times 2^{4} \times 2^{8} \times 2^{32}=2^{68}$ memory accesses, because for all of guessed $2^{24}$ keys in previous steps, we should guess 4 bits for $S K_{4}$ and for $2^{8}$ values encrypt $x_{3}$ one round and then update $N_{4}$ for $2^{32}$ times.

Step 7 requires $2^{28} \times 2^{12} \times 2^{32} \times 2^{4}=2^{72}$ memory accesses, because for all of guessed $2^{28}$ keys in previous steps, we should guess 12 bits for $S K_{22}$ and for $2^{32}$ values decrypt $x_{22}$ one round and then update $N_{5}$ for $2^{4}$ times.

Step 8 requires $2^{40} \times 2^{8} \times 2^{20} \times 2^{4}=2^{72}$ memory accesses, because for all of guessed $2^{40}$ keys in previous steps, we should guess 8 bits for $S K_{21}$ and for $2^{20}$ values decrypt $x_{21}$ one round and then update $N_{6}$ for $2^{4}$ times.

Step 9 requires $2^{48} \times 2^{4} \times 2^{12} \times 2^{4}=2^{68}$ memory accesses, because for all of guessed $2^{48}$ keys in previous steps, we should guess 4 bits for $S K_{20}$ and for $2^{12}$ values decrypt $x_{20}$ one round and then update $N_{7}$ for $2^{4}$ times.

Step 10 requires $2^{52} \times 2^{4} \times 2^{8} \times 2^{4}=2^{68}$ memory accesses, because for all of guessed $2^{52}$ keys in previous steps, we should guess $2^{4}$ for $S K_{19}$ and for $2^{8}$ values decrypt $x_{19}$ one round and then update $N_{8}$ for $2^{4}$ times.

Step 11 requires $2^{56} \times 2^{8}=2^{64}$ memory accesses, because for all of guessed $2^{56}$ keys in previous steps, we should read $2^{8}$ values of $N_{8}\left[x_{4}, x_{18}\right]$. 